\title{
Review of Time Series Analysis Method Research
}

\author{
Fei $\mathrm{Li}^{1, \mathrm{a}}$, Danyuan Zhao ${ }^{1, \mathrm{~b}}$,Muyang Wang ${ }^{1, \mathrm{c}}$,Xuan Wang ${ }^{1, \mathrm{~d}}$, Qiyue $\mathrm{Hu}^{1, \mathrm{e}}$ and \\ Chao Lv ${ }^{2, f}$ *
}

${ }^{1}$ School of Material Science and Engineering, Beijing Institute of Fashion Technology, Beijing 100029, China

${ }^{2}$ Computer Information Center, Beijing Institute of Fashion Technology, Beijing 100029, China

avooisabella@sina.com, b819816095@qq.com, '815468788@qq.com, ${ }^{\mathrm{d}} 759183711 @ q q . c o m$, emionai@yeah.net, Icrabbit@163.com

* please mark the corresponding author with an asterisk

Keywords: Time series; method; research

Abstract. Theory and method provided by time series analysis are one of the tools for large-scale high-difficulty comprehensive topic research. In recent years, many scholars obtained extremely fruitful results in research on time series. Some scholars developed new methods on the basis of existing time series analysis method. Innovation and development were searched in application. The author overviews and analyzes time series analysis method, thereby laying theoretical basis and reference basis for follow-up related study.

\section{Introduction}

Time series analysis is a branch of probability theory and mathematical statistics. Random data sequence is analyzed on the basis of probability statistics as theoretical basis. Mathematical model is established, namely model order determination and parameter estimation. The time series analysis is further applied in prediction, adaptive control, optimal filter and many other aspects.

Time series analysis method was originally started in 1927. In China, time series analysis was deeply studied and applied from late 1970s to medium and late 1980s. Its application field is wider and wider with more and more mature time series analysis method. Higher requirements are proposed on precision of model method. In recent years, many scholars obtained extremely fruitful results in the research on time series. Some scholars developed new methods on the basis of existing time series analysis method[1].

\section{Exponential smoothing method}

In recent years, time series prediction analysis method gets wide attention and research in the aspect of nonlinear model establishment and economic forecasting. Exponential smoothing method, as one of the important branches, becomes classic prediction and control model due to simple operation, strong applicability, excellent performance and wide application[2].

Exponential smoothing model was firstly proposed by C.C. Holt - a Canadian in unpublished report in 1957 for the first time[3]. Single exponential smoothing model is described in the report in details. Brown-an American described the method in details in his work in 1962. Brown high order exponential smoothing model was put forward, which was applied in prediction analysis of the nonlinear model[4]. In the late 1970s, Winter linear and seasonal index smoothing method appeared, therefore data seasonal and trend factors also can be included into consideration scope of index smoothing method[5,6]. In 1985, Gardner, E.S., Jr. (1985))-an American published an article for specifically discussing exponential smoothing method. The article still plays a very important role till present[7,8]. Then, exponential smoothing model is generalized to all fields of prediction science with very large effect. However, exponential smoothing model has the following three disadvantages: (1) it is difficult to determine smoothing initial value; (2) it is difficult for static smoothing parameter to adapt to own 
change of time series; (3) smooth parameter values are more dependent on people's experience, value determined in the method can not easily reach the best value[2].

\section{Cycle method}

There are many calculation methods for analyzing internal features of time series. Methods for analysis and detection include autocorrelation analysis, variance analysis, power spectrum analysis, filter analysis, stepwise regression analysis and other methods aiming at cycle features of time sequence.

In 2006, Xiao Zhiguo et al. combined with annual runoff, annual precipitation, annual rainfall in flood season and other hydrological data for analysis and comparison of three calculation methods in cycle analysis - simple partial wave method, Fourier analysis method and maximum entropy spectrum analysis method. Research results show that calculation result of simple partial wave method is strongly influenced by sample length. Results of Fourier analysis method and maximum entropy spectrum analysis method are relatively closer to overall truth values. However, the cycle analysis precision is lowered with increase of assumption sequence random components. Comprehensive analysis on actual sample calculation and statistical test shows that computational stability of maximum entropy spectrum analysis method is slightly better[9]. In 2007, Zhao Lihong and other people respectively studied five cycle analysis methods comparatively, namely simple partial wave method, Fourier analysis method, power spectrum analysis method, maximum entropy spectrum analysis method and wavelet analysis method. The results show that sequence period can be detected and analyzed by the five methods, thereby they can be used for cycle analysis. Calculation results of Fourier analysis method, maximum entropy spectrum analysis method and wavelet analysis method are more reliable. Sequence cycle values can be better detected, and they can be used as main methods for cycle analysis; Simple partial wave can produce some pseudo cycles; the power spectrum analysis method is greatly influenced by artificial factors. The results also showed that length of the time series can produce certain influence on cycle analysis calculation results. The sequence is longer, the calculation method is closer to sequence truth value[10].

\section{Time series analysis method of nonlinear dynamic system}

Chaos phenomenon is a widespread irregular movement in nature and society. It is a complex behavior generated by determined nonlinear dynamic system. Nonlinear time series analysis has become an important research hotspot in nonlinear information processing field in recent years with the deepening of the chaos theory and application technology research. It has more and more important application in related engineering field.

In 1980, Packard and other people proposed that strange attractor phase space can be reconstructed through nonlinear time series for the first time[11]. Thinking method of nonlinear dynamic characteristics was studied. Complex chaotic movement prologue of dynamic system was studied through scalar time series. Kens independently proposed that strange attractor phase space is constructed by scalar time series in delay coordinate method[12]. One-to-one corresponding famous embedding theorem of original system and reconstruction system is proved. In 1983, Grassberger and Procaccia published thesis of scalar strange attractor singularity (chaos degree) in physics, and they put forward a famous GP algorithm of calculating correlation dimension[13]. In 1988, Cvitanovic pointed out: chaos skeleton is composed by infinite unstable periodic orbit. The important progress on recognition allows people not to comprehend it from general nature from chaos movement. It can be comprehended and grasped from chaotic internal structure[14]. In 1992, Theiler and other people proposed concept method for replacing data creatively on the basis of summarizing previous work from nonlinearity of detection time sequence[15]. In 1996, Barahona and Poon published thesis of prediction result nonlinear detection on Nature. Non-linear determination components in time sequence are tested according to prediction effect[16]. In 2000, Hegger, etc. wrote thesis and firstly proposed excessive embedding method aiming at non-stationary signal on Physical Review Letters[17]. In 2006, Veraes etc. proposed modeling and prediction method of non-stationary system 
on the basis of the above method[18]. In recent years, a large number of researchers adopted nonlinear model, especially neural network model and support vector machine model (SVM) for global or local modeling and forecasting aiming at nonlinear time series. General method provided by nonlinear time series and its latest achievements will drive related discipline research to make progress. The progress is likely to be a breakthrough. Therefore, nonlinear time series analysis has always been the most active area of nonlinear dynamics since its generation.

\section{Multi-scale analysis method}

Many phenomena and processes have multi-scale characteristics or multi-scale effect in nature and engineering practice. Meanwhile, people observe and measure phenomenon or process in different scales. Therefore, it is very natural to apply multi-scale system theory for describing and analyzing these phenomena or processes. It can better manifest essential characteristics of these phenomena or processes. The application of multi-scale idea to time series analysis is regarded as multi-scale time series analysis.

Wang Songwei and other people developed and studied several basic problems and methods in time series analysis within multi-scale framework, namely second moment analysis, maximum likelihood estimation of time series model parameter and real-time dynamic forecast of time sequence. Traditional time series analysis method is enriched. Basic attributes of multi-scale variance is studied. Estimation method of multi-scale variance and estimate value confidence interval algorithm are provided. In addition, the multi-scale variance is applied to estimation of time series spectrum density function as an application. Decorrelation property of wavelet transform is utilized for respectively developing multi-scale maximum likelihood estimation method based on discrete wavelet transform and discrete wavelet packet transform. Problem of large calculation amount in traditional likelihood function estimation is effectively lowered. Economic, financial and other environments have a lot of cyclical non-stationary time series. Kalman filtering method and multi-scale analysis method are combined for proposing real-time and recursive expansion wavelet-Kalman filter mixed estimation and prediction methods with multi-scale analysis ability[19].

\section{Conclusion}

General method provided by time series analysis and its latest achievements will push related discipline research to make progress. A large number of excellent scientific research personnel provide a steady stream of creative thinking for analyzing and dealing with time series problem. Research in the field keeps rapid development momentum. Meanwhile, many discipline fields are closely combined for providing time series analysis with wide development space and rich research contents.

\section{Acknowledgments}

The paper is supported by Beijing Institute of Fashion Technology 2015 undergraduate scientific research training project 'Time Series Analysis and Its Application Research'.

\section{References}

[1] Y.Tang: The Research and Application of The Time Series Analysis, M.S.thesis, Northeast Agrieultural University, China, 2007.

[2] L.Qi: Research of The Decision Support Algorithm Based on Exponential Smoothing of Time Series, M.S.thesis, Yanshan University, China, 2007.

[3] Holt.C.Foreeasting Seasonal and Trends by ExPonentially Weighted Moving Averages. PA7 Camegie Institute of Technology Available from the Engineering Library, University of Texas at Austin, International Journal of Foreeasting, 1957,52(1):5-13. 
[4] Brown, Goodell. Smoothing Forceasting and Prediction of Dicerete Time Series. Englewood Cliffs, NJ7 Prentiee--Hall, 1963:3-24.

[5] Winters.R. Forceasting Sales By Exponeniially Weighted Moving Averages. Management Science, 1970,6(3):324-342.

[6] Holt.C. Forceasting Seasonals and Trends by Exponeniially Weighted Moving Averages. Iniernational Journal of Forecasting, 2004, 20(1): 5-10.

[7] Gardner.Jr. Exponential Smoothing: The State of the Art.Journal of Forceasting, 1985,4(4):1—28.

[8] Everette.S, Gardner.Jr. Exponential Smoothing: The State of the Art-Part Two. International Journal of Forceasting, 2006, 15(1):637-666.

[9] Z.G.Xiao: Comparison of Different Analysis Methods for the Periodicity of Hydrological Time Series, M.S.thesis, College of Water Resource and Environment, Hohai University, China ,2006.

[10] L.H.Zhao: Study of Analysis Methods for the Period of Hydrologieal Time Series , M.S.thesis, College of Water Resource and Environment, Hohai University, China ,2007.

[11] N.H.Packard,J.P.Crutchfield, J.D.Fanners,etal. Geometry from a time series.Phys Rev Lett, 1980,45:712-716.

[12] F.Takens. Deteeting strange attractors in fluld turbulence.D.Rand.L.S.Young. Dynamical systems and turbulence.Berlin: Springer, 1981:366-381.

[13] P. Grassberger I. Procaeeia, Measuring the strangeness of strange Attraetors. Physica D, 1983,9:198-208.

[14] P.Cvitanovie. Invariant measure ment of strange sets in terms of cycle. Phys.Rev.Lett., 1988,61:27-29.

[15] J.Theiler etal . Testing for nonlinearity in time series: The methed of sunrogate data. Physica $\mathrm{D}, 1992,58: 77$.

[16] M.Barahona,C.--S. Poon. Deteetion of nonlinear dynamics in short, noisy time series.Nature, 1996,381:215-217.

[17] R.Hegger,H.Kantz, L. Matassini, and T.Sehreiber. CoPing with nonstationarity by overembedding. Phys.Rev;Lett.,2000,84(18):4092—40095.

[18] P.F.Verdes, P. M. Granitto, and H.A.Ceccatto. Overelnbedding Method for Modeling Nonstationary Systems. Phys.Rev.Lett.,2006,96(11):118701.

[19] S.W.Wang: Researches on the Multi-Scale Time Series Analysis, M.S.thesis, Henan University, China ,2006. 